TPeriodica Polytechnica Electrical Engineering and Computer Science

\author{
61(2), pp. 193-197, 2017 \\ https://doi.org/10.3311/PPee.9982 \\ Creative Commons Attribution (1)
}

RESEARCH ARTICLE

\section{On the Graphicity of the Independence Structure of Linear Active Networks}

\author{
Csongor Gy. Csehi ${ }^{1 *}$, András Recski ${ }^{1}$
}

Received 05 September 2016; accepted 22 April 2017

\begin{abstract}
Consider a linear network composed of 2-terminal devices. Its interconnection structure is described by a graph $G$. The voltages or the currents of a subset of devices can independently be prescribed if and only if the subset of the corresponding edges in the graph $G$ is circuit-free or cut set free, respectively. This classical result of Kirchhoff can be generalized for networks containing multiterminal devices as well: the independence structure can be described by the circuits and cut sets of a more general abstract mathematical structure, a matroid M. However, these matroids will not always be graphic. Using some recent mathematical results for characterizing graphic structures among the matroids, here we give a physical characterization of subclasses of those active networks where $M$ happens to be graphic.
\end{abstract}

\section{Keywords}

matroid theory, linear network, multiterminal devices

\section{Introduction}

Electric network analysis was the first real application of graph theory, almost 170 years ago. The laws of Kirchhoff [1] related the voltages and the currents of the devices to the circuits and cut sets, respectively, of the graph of the interconnection.

These classical results can be applied if the network consists of 2-terminal devices only. Let us consider the interconnection of various linear multiports leading to a linear network. If the multiterminal devices are modelled by controlled sources then the interconnection can still be described by a graph but, due to the controls among the edges, the independence structure of the network will not always remain graphic. Since the network is linear, it can be described by a matrix and there is a mathematical tool, matroid theory, to describe the combinatorial properties of the independence structure of the columns of this matrix but these matroids will rarely be graphic (that is, the cycle matroids $M(G)$ of some graph $G)$.

The matroid operation union (also called sum) turned out to be the appropriate tool to describe the effect of control, as found independently by [2,3] and [4]. However, the subset of graphic matroids is not closed with respect to union, in fact, the union of two graphic matroids is often outside the more general subset of binary matroids.

The fundamental results of [5] and [6] characterize those graphic matroids whose union is the free matroid (the cycle matroid of a tree). If the union of several copies of the same graphic matroid is considered then one can decide if this union is graphic [7] but the question is still open for general addends. A possible approach is to fix a graph $G_{0}$ or its cycle matroid $M_{0}=M\left(G_{0}\right)$ and study those graphs $G$ where the union of $M(G)$ and $M_{0}$ is graphic. If $M_{0}$ consists of loops only or it contains bridges then the problem is trivial hence the first interesting question was if $G_{0}$ consists of a circuit of length two (two parallel edges) and any number of loops. In the language of electric network analysis this corresponds to the linear active networks composed of 2-terminal passive devices plus a single current controlled current source. This case has been solved in [8] - mathematically it was a Kuratowski-type characterization of $G$ which had a physical interpretation as the lack of feedback, see Theorems A through D below.

\footnotetext{
${ }^{1}$ Department of Computer Science and Information Theory, Faculty of Electrical Engineering and Informatics, Budapest University of Techology and Economics, H-1521 Budapest, P.O.B. 91, Hungary

*Corresponding author, e-mail: cscsgy@cs.bme.hu
} 
The results of [8] have recently been generalized for the case if $G_{0}$ consists of either $n$ series or $n$ parallel edges in addition to the loops, see [9] for $n=3$ and [10] for any $n$. In the present paper we study the interpretation of the structure of $G_{0}$ in terms of controlled sources, and formulate the mathematical meaning of these recent results in the language of electric network analysis.

\section{Former Results}

Throughout, we use the notation of [11]. Suppose that a network is composed of 2-terminal devices and current controlled current sources (CCCS). The graph of the network is defined in the usual way (each CCCS corresponds to a pair of edges), and we assign orientation to each edge arbitrarily. There are several equations among the currents of the devices, some of them are the Kirchhoff Current Laws, describing the topology of the network, some others describe the controls. In what follows, we shall refer to these sets of equations as the graphic and the algebraic sets of equations, respectively. For example, the graph of the network of Fig. 1a is shown in Fig. 1b, the set of the graphic equations consists of

$$
\begin{aligned}
& i_{1}+i_{2}+i_{3}=0 \\
& i_{3}-i_{4}-i_{5}=0
\end{aligned}
$$

(and any linear combinations of them), while there is a single algebraic equation

$$
i_{5}=c \cdot i_{2}
$$

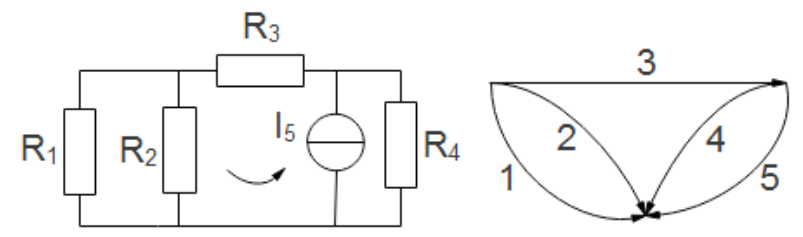

Fig. 1 A network and the corresponding graph

Hence there are three linear equations referring to the five currents and these equations can be summarized by the coefficient matrix

$$
M_{1}=\left(\begin{array}{ccccc}
1 & 1 & 1 & 0 & 0 \\
0 & 0 & 1 & -1 & -1 \\
0 & c & 0 & 0 & -1
\end{array}\right)
$$

In contrast, the network of Fig. 2 has a different kind of control, namely $i_{5}=c \cdot i_{3}$, hence our matrix will be

$$
M_{2}=\left(\begin{array}{ccccc}
1 & 1 & 1 & 0 & 0 \\
0 & 0 & 1 & -1 & -1 \\
0 & 0 & c & 0 & -1
\end{array}\right)
$$

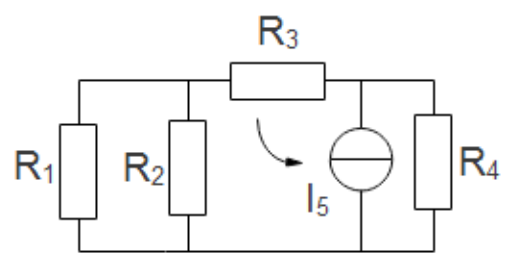

Fig. 2 A network, similar to that of Fig. 1 but with a different kind of control

If the five column vectors of the matrices are considered then in case of $\mathbf{M}_{1}$, any three out of the five vectors except $\{1,3,4\}$ are linearly independent" while in case of $\mathbf{M}_{2}$, , a set of three vectors are linearly independent if and only if the set does not contain both the first and the second vectors." (In the first case we suppose $c \neq 0, c \neq-1$ and in the second case we suppose that $c \neq 0, c \neq 1$, see the remark on genericity at the end of the paper as well.)

In the second case the italicized sentence can be rephrased as follows: ,, a set of three vectors are linearly independent if and only if the corresponding three edges form a spanning tree in the graph of Figure 3. " On the other hand, no such reformulation is possible in the first case - no one can draw a graph with four vertices and five edges so that $\{1,3,4\}$ is a circuit and any other set of three edges forms a spanning tree.

Using the terminology of matroid theory we may conclude that the matroid describing the second network is graphic while that for the first one is non-graphic.

In both cases the first two rows of the matrices refer to the graphic set of equations and the last row refers to the algebraic one. This partition of the rows leads to a graphic and to an algebraic submatrix. One can easily see that a subset of columns of both graphic submatrices is linearly independent if an only if the corresponding edges form a circuit-free subgraph of the graph of Fig. 4. On the other hand, such a subset of columns of the algebraic submatrix of the first and the second network is linearly independent if and only if the corresponding edge set is circuit-free in the graphs of Fig. 5 and 6, respectively.

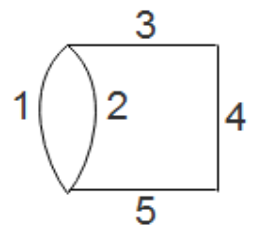

Fig. 3 A graph representing $\mathrm{M}_{2}$

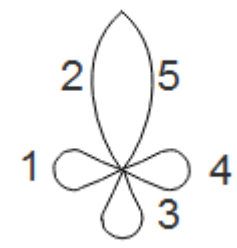

Fig. 5 The independence structure of the control of Fig. 1
3

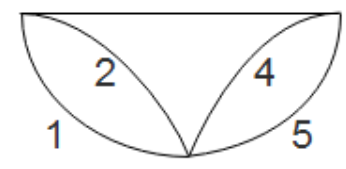

Fig. 4 The graph representing the interconnection of both networks

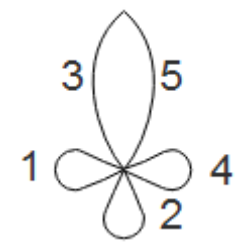

Fig. 6 The independence structure of the control of Fig. 2 
Needless to say, this last sentence is unnecessarily complicated - for example, instead of saying that a subgraph is circuit-free in Fig. 5 one rather says that only the second or the fifth element is permitted but not both. However, we wanted to illustrate that the independence structures could, in this case, be illustrated by a graph as well.

The above examples illustrate the necessity of the condition in the following theorem:

Theorem A [8]: Let $G_{0}$ consist of a circuit of length two (two parallel edges $a, b)$ and any number of loops. Let $M_{0}$ denote the cycle matroid $M\left(G_{0}\right)$. Let $G$ be an arbitrary graph on the same edge-set. Then the union of $M(G)$ and $M_{0}$ is graphic if and only if $G$ does not contain any subgraph isomorphic to the graph of Fig. 7 or to its subdivision, with $a$ and $b$ in the indicated positions.

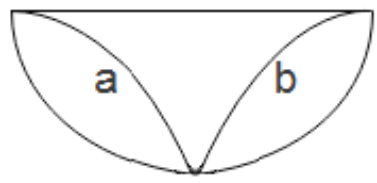

Fig. 7 The graph whose existence characterizes the presence of feedback

If a network is composed from 2-terminal devices and of a single CCCS (whose edges will play the role of $a$ and $b$ ) then the existence of the subgraph of Fig. 7 or its subdivision (with $a$ and $b$ in the requested positions) means the presence of a feedback $F$, no matter what kind of subnetworks $N_{1}, N_{2}$ are interconnected, see Fig. 8. Hence the above theorem can be reformulated as follows:

Theorem B [8]: Suppose that a network is composed of 2-terminal devices and of a single current controlled current source. The independence structure describing the currents of the devices is graphic if and only if there is no feedback in the network.

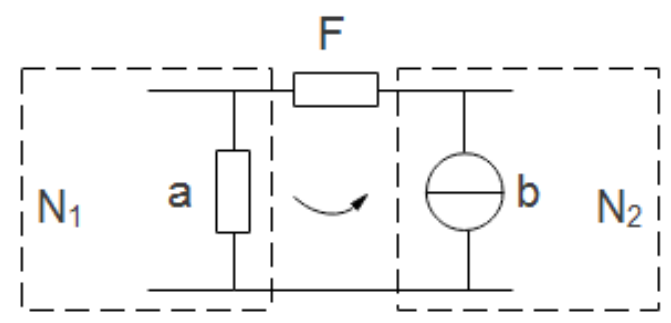

Fig. 8 Feedback in a general network

The graph $G$ in Theorem A was arbitrary. In network theory applications we may always suppose that the underlying graph of the electric network is connected, in fact, even 2-connected if there is no control in the network. Moreover, if a subgraph is connected along two points to the rest of the graph and none of the edges of this subgraph is a controlling or a controlled element then the whole subgraph can be replaced by a single edge. Using these replacements if applicable, we obtain the reduced graph of the network. For a more formal description of this matroid theoretical reduction see Section 2 of [10].

In view of this, feedback is formally defined as the presence of at least one circuit in the complement of $\{a, b\}$ in the reduced network graph. Then one can reformulate Thorem A as follows:

Theorem C: Suppose that the reduced graph of the network is 2-connected and $a, b$ are two non-serial edges. Then there is a subgraph isomorphic to Fig. 7 or its subdivision, with $a$ and $b$ in the specified positions, if and only if the complement of $\{a, b\}$ in the reduced network graph contains at least one circuit.

In the next section we shall refer to the negative of this reformulation:

Theorem D: Suppose that a network is composed of 2-terminal devices and of a single current controlled current source involving the edges $a, b$. We may suppose without loss of generality that the reduced graph of the network is 2-connected and $a, b$ are two non-serial edges. Then the independence structure describing the currents of the devices is graphic if and only if there is no feedback in the network, that is, if the complement of $\{a, b\}$ in the reduced network graph is circuit-free.

In what follows we shall generalize Theorems B and D for more general types of control. Recall that in case of a CCCS the current of a single source is controlled by the current of a single resistor. We have found analogous results if only one of these restrictions remains.

\section{New Results, Part One \\ 3.1 Several Controlled Sources and a Single Controlling Element}

Suppose that the current of a single resistor $R_{0}$ controls several current sources $I_{1}, I_{2}, \ldots, I_{k}$ as described by the respective equations $i_{j}=c_{j} \cdot i_{0}$ for every $j=1,2, \ldots, k$. We may suppose that the set $[n]$ of the corresponding edges $e_{0}, e_{1}, e_{2} \ldots, e_{k}$ does not contain any cut-set in the graph of the network, since otherwise there were an additional equation $\Sigma \pm i_{j}=0$ among some of these currents, which, together with the control equations $i_{j}=c_{j} \cdot i_{0}$, would lead to a singular network.

Since there are $k$ controls in the network, the above definition of the feedback is modified as the presence of at least one circuit in the complement of the set $[n]$ in the reduced network graph. 
Theorem 1. Suppose that a network is composed of 2-terminal devices and the current of a resistor $R_{0}$ controls several current sources $I_{1}, I_{2}, \ldots, I_{k}$ as described by the respective equations $i_{j}=c_{j} \cdot i_{0}$ for every $j=1,2, \ldots, k$ (where the control constants $c_{1}, c_{2} \ldots, c_{k}$ are generic parameters, that is, they are algebraically independent over the field of the rational numbers). We may suppose without loss of generality that the above set $[n]$ is cut set free. Then the independence structure describing the currents of the devices is graphic if and only if there is no feedback in the network, that is, if the complement of $[n]$ in the reduced network graph is circuit-free.

Proof: The system of equations $i_{j}=c_{j} \cdot i_{0}$ for every $j=1,2, \ldots, k$ leads to an algebraic submatrix representing a matroid $M_{1}$ which consists of loops and a single circuit of length $k+1$. Let $M_{2}$ denote the matroid, represented by the graph of the interconnetion. Proposition 14 of [10] states that the union of the reduced matroids $M_{1}$ ' and $M_{2}$ ' is graphic if and only if either [n] contains a cut-set or $M_{2}{ }^{\prime} \backslash[n]$ is the free matroid. Since the former case is excluded, the reduced network graph without the edges in $[n]$ must be circuit-free. $\square$

\subsection{Several Controlling Elements and a Single Controlled Source}

Suppose that a single current source $I_{0}$ is controlled by the current of several resistors $R_{1}, R_{2}, \ldots, R_{k}$ as described by the equation $i_{0}=\Sigma c_{j} \cdot i_{j}$ where the summation is for every $j=$ $1,2, \ldots, k$. We may suppose without loss of generality that the network graph is either 2-connected or the set $[n]$ of the corresponding edges $e_{0}, e_{1}, e_{2}, \ldots, e_{k}$ has at least one edge from each 2-connected component.

Since there is a single control involving $k+1$ elements in the network, the above definition of the feedback is modified as the presence of at least one circuit in the complement of any two-element subset of the set $[n]$ in the reduced network graph.

Theorem 2. Suppose that a single current source $I_{0}$ is controlled by the current of several resistors $R_{1}, R_{2}, \ldots, R_{k}$ as described by the equation $i_{0}=\Sigma c_{j} \cdot i_{j}$ where the summation is for every $j=1,2, \ldots, k$. Like in Theorem 1 , we suppose that the control constants $c_{1}, c_{2} \ldots, c_{k}$ are generic parameters, that is, they are algebraically independent over the field of the rational numbers. We may suppose without loss of generality that the network graph is either 2-connected or the set $[n]$ of the corresponding edges $e_{0}, e_{1}, e_{2} \ldots, e_{k}$ has at least one edge from each 2-connected component. Then the independence structure describing the currents of the devices is graphic if and only if there is no feedback in the network, that is, if the complement of the edge set $\{a, b\}$ is circuit-free for any two non-serial edges $a, b$ of $[n]$ in the same 2-connected component of the reduced network graph.
Proof: The equation $\boldsymbol{i}_{\mathbf{0}}=\boldsymbol{\Sigma} \boldsymbol{c}_{\boldsymbol{j}} \cdot \boldsymbol{i}_{\boldsymbol{j}}$ leads to an algebraic submatrix representing a matroid $\boldsymbol{M}_{\mathbf{1}}$ which consists of loops and $\boldsymbol{k}+\mathbf{1}$ parallel edges. Proposition 22 of [10] states that the union of the reduced matroids $\boldsymbol{M}_{1}{ }^{\prime}$ and $\boldsymbol{M}_{2}{ }^{\prime}$ is graphic if and only if no 2-connected component of the reduced network graph $\boldsymbol{G}$ has two non-serial edges $\boldsymbol{a}, \boldsymbol{b}$ so that $\boldsymbol{G}-\{\boldsymbol{a}, \boldsymbol{b}\}$ contains a circuit. This is clearly equivalent to the condition of Theorem 2 . $\square$

\section{Examples and a Remark}

Example 1. Consider the network of Fig. 9 where $i_{0}=c_{1} \cdot i_{1}+$ $c_{2} \cdot i_{2}$. The graph of the network is given in Fig. 10. The coefficient matrix for the system of equations for the currents of the elements will be

$$
\left(\begin{array}{llllll}
-1 & c_{1} & c_{2} & 0 & 0 & 0 \\
1 & 1 & 0 & 1 & 0 & 0 \\
1 & 0 & 0 & 0 & 1 & 0 \\
0 & 0 & 1 & 0 & 0 & 1
\end{array}\right)
$$

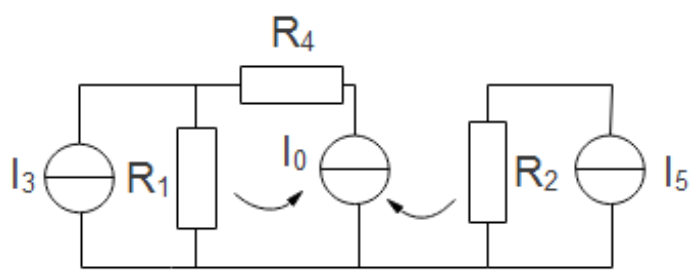

Fig. 9 The network of Example 1

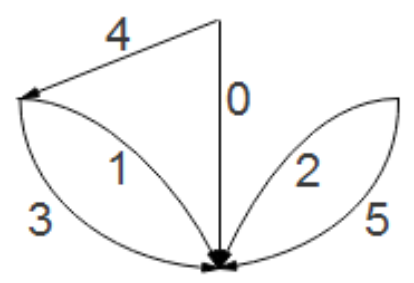

Fig. 10 The graph of the network of Example 1

The matroid represented by the columns of this matrix has six elements and rank four. This matroid is non-graphic - if we contract elements 4 and 5 then the resulting minor is the rank 2 uniform matroid on the set $\{0,1,2,3\}$ which is known not to be binary, let alone graphic. Based on Theorem 2 one could reach the same conclusion: The elements 0 and 1 are non-serial edges in the same 2-connected component of the graph of Fig. 10, still the complement of the set $\{0,1\}$ contains a circuit, namely $\{2,5\}$.

Example 2. The network of Fig. 11 illustrates Theorem 1. Let the controls be $i_{1}=c_{1} \cdot i_{0}$ and $i_{2}=c_{2} \cdot i_{0}$. The graph of the network is given in Fig. 12 and the coefficient matrix for the system of equations for the currents of the elements will be 


$$
\left(\begin{array}{lllllll}
c_{1} & -1 & 0 & 0 & 0 & 0 & 0 \\
c_{2} & 0 & -1 & 0 & 0 & 0 & 0 \\
1 & 1 & 0 & 1 & 1 & 0 & 0 \\
0 & 1 & -1 & 0 & 0 & 0 & 1 \\
0 & 0 & 0 & 0 & -1 & 1 & 1
\end{array}\right)
$$

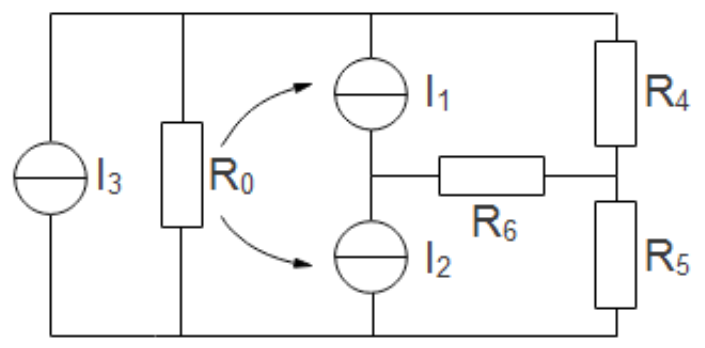

Fig. 11 The network of Example 2

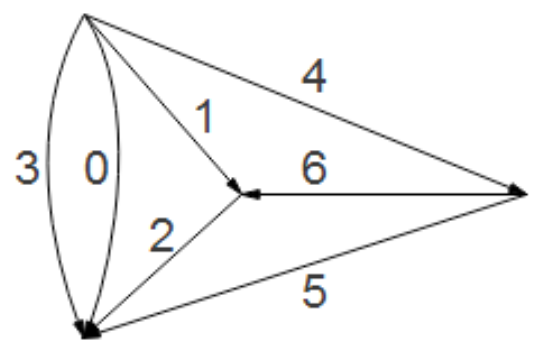

Fig. 12 The graph of the network of Example 2

The corresponding matroid has seven elements and rank five. One can see that it is non-graphic - if we contract elements 0,2 and 6 , the resulting minor is the rank 2 uniform matroid on the set of the remaining elements. Based on Theorem 1 one could reach the same conclusion: If we delete the edges of the set $[n]=\{0,1,2\}$ from the graph of Fig. 12, the remaining graph contains a circuit, namely $\{3,4,5\}$.

Remark: Results applying matroid union for engineering applications frequently require a genericity-type condition like the one we had in Theorems 1 and 2 concerning the control constants $c_{1}, c_{2} \ldots, c_{k}$. The basic reason of this has been discovered by Edmonds [12] during his study about the relation between rank and term rank of the matrices. If such an assumption is missing, the statement might be wrong.

For example, suppose that $c_{1}=-1$ in Example 1. Then the set $\{0,1,4\}$ will become a circuit and the matroid will be graphic (a circuit formed by $\{0,1,4\}$ and another formed by $\{1,2,3,5\}$, sharing a common edge). Physically, it corresponds to a singular network: The relation $c_{1}=-1$ leads to a control equation $i_{0}=-i_{1}+c_{2} \cdot i_{2}$; hence the Kirchhoff equation $i_{3}=-\left(i_{1}+i_{0}\right)$ would lead to a relation $i_{3}=c_{2} \cdot i_{5}$ between two independent current sources.

\section{Acknowledgement}

Our research has been supported by the grant \# OTKA 108947 of the Hungarian National Research, Development and Innovation Office (NKFIH).

\section{References}

[1] Kirchhoff, G. "Über die Auflösung der Gleichungen, auf welche man bei der Untersuchung der linearen Verteilung galvanischer Ströme geführt wird." Annalen der Physik. 148(12), pp. 497-508. 1847. (in German) https://doi.org/10.1002/andp.18471481202

[2] Recski, A. "On partitional matroids with applications." Colloquia Mathematica Societatis Janos Bolyai. 10, pp. 1169-1179. 1973.

[3] Iri, M., Tomizawa, N. "A unifying approach to fundamental problems in network theory by means of matroids." Transactions of the Institute of Electronics and Communication Engineers of Japan. 57A(8), pp. 35-41. 1975.

[4] Narayanan, J. "Theory of Matroids and Network Analysis." Ph.D. Thesis in Electrical Engineering, Indian Institute of Technology, Bombay, India, 1974.

[5] Nash-Williams, C. St. J. A. "Decomposition of finite graphs into forests." Journal of the London Mathematical Society. s1-39(1), p. 12. 1964. https://doi.org/10.1112/j1ms/s1-39.1.12

[6] Edmonds, J. "Minimum partition of a matroid into independent subsets." Journal of Research of the National Bureau of Standards. 69B, pp. 67-72. 1965.

[7] Lovász, L., Recski, A. "On the sum of matroids." Acta Mathematica Academiae Seientiarum Hungaricae. 24(3-4), pp. 329-333. 1973.

[8] Recski, A." On the sum of matroids II." In: Proceedings of the Fifth British Combinatorial Conference. University of Aberdeen, Aberdeen, July 14-18, 1975. pp. 515-520.

[9] Csehi, Cs. Gy. "Matroidelméleti vizsgálatok és alkalmazásaik." Master's Thesis, Budapest University of Technology and Economics. 2012. (in Hungarian)

[10] Csehi, Cs. Gy., Recski, A. "The graphicity of the union of graphic matroids." European Journal of Combinatorics. 50, pp. 38-47. 2015. http://doi.org/10.1016/j.ejc.2015.03.022

[11] Recski, A. "Matroid Theory and its Applications in Electric Network Theory and in Statics." Springer, Berlin, 1989.

[12] Edmonds, J. "Systems of distinct representatives and linear algebra." Journal of Research of the National Bureau of Standards - B. Mathematics and Mathematical Physics. 71B(4), pp. 241-245. 1967. 